\title{
Germanica
}

GERMANICA

$53 \mid 2013$

Littérature et cinéma dans l'espace germanophone contemporain : jeux intermédiaux, modes de transfert, adaptations

\section{Les nouvelles conjonctions d'images et de lettres chez Alexander Kluge}

Neue Konjunktionen von Bildern und Buchstaben bei Alexander Kluge

New Conjunctions of Letters and Images in the Work of Alexander Kluge

Herbert Holl

\section{OpenEdition}

Journals

Édition électronique

URL : http://journals.openedition.org/germanica/2300

DOI : $10.4000 /$ germanica. 2300

ISSN : 2107-0784

Éditeur

Université de Lille

Édition imprimée

Date de publication : 30 décembre 2013

Pagination : 275-289

ISBN : 9782913857322

ISSN : 0984-2632

Référence électronique

Herbert Holl, «Les nouvelles conjonctions d'images et de lettres chez Alexander Kluge », Germanica

[En ligne], 53 | 2013, mis en ligne le 30 décembre 2016, consulté le 06 octobre 2020. URL : http://

journals.openedition.org/germanica/2300 ; DOI : https://doi.org/10.4000/germanica.2300

(c) Tous droits réservés 


\title{
Les nouvelles conjonctions d'images et de lettres chez Alexander Kluge
}

\author{
Herbert HOLL \\ Université de Nantes
}

Au commencement, l'imagicité, c'est la fusion du mot et de l'image, la consanguinité de la littérature et du cinéma - à travers le « langage gestuel intensifié » du cinéma ${ }^{1}$. D'après Eisenstein, cette imagicité est la « source productive du sens », qui précipite mouvement, émotion et perception. Chez Alexander Kluge, récit, image, concept se tissent en contexture de puissances de différenciations et de sentiments ${ }^{2}$. À la fin, images et lettres se jettent les unes dans les autres en kaléidoscope, en poétique du « vide quantique » - « la poésie, c'est le réel authentiquement absolu », « die Poesie ist das echt absolut Reelle », aurait dit Novalis ${ }^{3}$. Or, la mécanique quantique manifeste ce principe d'une littérature d'incertitude, d'une image-mouvement, tel Friedrich Kittler, qui, dans « Musique et mathématique », avait constellé l'image d'un brin de bruyère simulé mathématiquement avec son programme informatique. Par là, il abolissait, poétisait, tels Kluge et Eisenstein, les lignes de séparation entre la mechanè et la technique. Kluge serait alors plus

1. - Georges Didi-Huberman, « Aby Warburg et l'archive des intensités », in : Études photographiques $\mathrm{n}^{\circ} 10,2001$, p. 144-168, http://etudesphotographiques.revues. org/index268.html, p. 4 (consulté le 24 juin 2011).

2. - Cf. Herbert Holl, La fuite du temps. «Zeitentzug » chez. Alexander Kluge. Récit. Image. Concept, Berlin - Bern - New York e. a., Peter Lang, 1999.

3. - Alexander Kluge, Chronik der Gefühle, t. 2, Frankfurt a.M., Suhrkamp, 2000, p. 977.

GERMANICA, 2013, LIII, p. 275 à 289. 
proche que jamais de Friedrich Kittler, qui a légué avant sa mort ses ordinateurs au Literaturarchiv de Marbach ${ }^{4}$.

\section{Hortus conclusus}

S'en retourner, c'est en réchapper. Le film Heimkehr de Gustav Ucicky, qui ouvre dans le cinéma initiatique de Kluge la " nouvelle histoire » du raid aérien sur Halberstadt le 8 avril 1945 dans l'Inquiétance du temps de $1977^{5}$, téléporte, de Kairos-Film dans la Friedrichstraße à Munich, jusque dans le res nullius cosmique d'internet, en passant par la DCTP de Düsseldorf, le Capitol de Halberstadt, sa grande salle paradigmatique, aux fauteuils de velours rouge d'antan où l'on donnait dans l'ordre les actualités, le documentaire et le film, séparés par les esquimaux de l'entracte.

Le jardin-internet, à la fois enclos et déclos, umgrenzt, entgrenzt, règne chez le Kluge tardif. C'est celui du rhizome, des choses en leurs mondes, des Menschenkinder en leurs têtes. Depuis toujours, mais surtout après le 11 septembre 2001, tous les films instituent un seul jardin infini auquel reviennent, en provenance de l'avenir, «toutes les formes précoces du récit-image », Bilderzählung, les débuts de l'histoire du cinéma, des histoires brèves, laconiques - « chaque histoire une métaphore ». Ce sont des histoires-minutes, histoires-décennies, histoires-siècles, histoires-millénaires ${ }^{6}$. Tel serait le jardin dompté, domestiqué, en quelque sorte le «parc humain » de Peter Sloterdijk avec sa trouée temporelle laissée par le diable, mais dé-démonisée, de seconde nature, une « enclave hétérotopique ». C'est là l'heure d'Internet : «Créer des jardins dans le tsunami des données et par là, les préserver d'elles-mêmes ». Kluge en supprime autant qu'il en recueille, au cour de cette « anti-nature de la toile » dont la surpuissance même auto-génère la résistance contre elle ${ }^{7}$. Après le célèbre fichier de Niklas Luhmann, où rien ne se déplaçait, ne se déclassait, un nouveau médium

4. - Morten Freidel, « Poesie der Technik. Das Marbacher Archiv zeigt Kittlers Computer », in : FAZ, 19. Juni 2013, p. $4:$ « auch technische Maßstäbe an Literatur anlegen $[\ldots] »$. Il s'agit d'un manuscrit inédit de Friedrich Kittler.

5. - Deuxième cahier d'Unheimlichkeit der Zeit, Der Luftangriff auf Halberstadt am 8. April 1945, repris dans la Chronik der Gefühle, op. cit., t. 1, ici : p. 27.

6. - « Wir leben doch nicht nur in einer Gegenwart », entretien de Jochen Rack avec Alexander Kluge, in : Sinn und Form, n 4, 2008, p. 471-481, cit. p. 477 : « kurze, lakonische Geschichten, jede Geschichte eine Metapher. »

7. - Alexander Kluge, in: FAZ 14.01.2010 «Gärten anlegen im Daten-Tsunami », Ein Gespräch mit Uwe Ebbinghaus, consultable en ligne: http://www.faz.net/aktuell/ feuilleton/debatten/digitales-denken/alexander-kluge-gaerten-anlegen-im-datentsunami-1596476.html. $C f$. également d'Alexander Kluge, « Gärten sind wie Brunnen. Im Innern eines jeden ernsten Menschen - auch wenn er spielt - findet sich ein , geschlossener Garten' », in Text + Kritik, n 85/86, édition refondue, novembre 2011, p. 5-8. 
nuvolaire débordant internet, télévision, espace public, livre, les relie tous in nuce. Alors se connectent, selon Kluge, «les petites créatures, non par les grandes, et l'internet est le forum des petites. » Dans ce jardin, la dé-limitation médiale reçoit le livre, pourtant circonscrit par sa matérialité de papier.

Or, après la loge secrète du Capitol d'enfance de Kluge, le hortus conclusus se peuple à présent des réceptacles, Gefäße, qui contenaient déjà dans les entretiens entre Kluge et Heiner Müller l'infinitude de la métaphore. Ces vases s'évasent en une "dramaturgie constellante », konstellative Dramaturgie, mêlant la littérature à celle de la physique et de la cinématographie. Dans Le Labyrinthe de la tendre force, sous la constellation des Pléiades du livre ouvert de Galilée, Sternenbotschaft apparait sur la page de droite, le cosmos et l'amour soumis à une « singulière espèce d'attraction », illustrée par un fragment de roman d'Edgar Allan Poe sur la page de gauche :

Il s'agirait plutôt (tel serait le principe de son œuvre [Balzac]) de parvenir par coordination à une cartographie intégrale de l'Histoire, un texte dont chaque chapitre soit un roman, et chaque roman une histoire épocale. Ce serait là le principe de la constellation libre et mobile, tels les corps célestes en gravitation les uns autour des autres. [...]. Cent ans plus tard, Eisenstein adopte ce principe narratif, proposant une dramaturgie sphérique ainsi que des livres-sphères : autour d'un même centre circulent à la surface de la sphère, mais mues par l'attraction du centre, les diverses histoires. Il n'y en a pas moins dialectique, affirme Eisenstein en référence aux auteurs du Talmud de Babylone, entre la surface et le noyau. La surface serait en même temps le noyau. Mais le noyau s'avérerait, dès lors qu'on le met en récit, c.-à-d. le déploie, comme surface profuse ${ }^{8}$.

Cette singulière attraction qu'exerce la force poétique sur les jardins, nids et puits d'amour exige que le jardin s'enfonce dans ce Labyrinthe de la tendre force aux cent-soixante-six variations, en résonance avec des images mues, des films et des écrits se mouvant en musique. Celle-là même qui fait apparaître les galaxies en lieux fictifs ${ }^{9}$. Edgar Reitz situe son compagnon d'armes dans cette même constellation : « Alexander Kluge agit dans la marge, appartient pourtant entièrement au centre du cinéma allemand ${ }^{10}$. » Mais y a-t-il un centre?

8. - Alexander Kluge, Das Labyrinth der zärtlichen Kraft. 166 Liebesgeschichten, Frankfurt a.M., Suhrkamp, 2009, p. 339 sq. Traduction inédite de Kza Han et Herbert Holl, à paraître aux Presses Universitaires Blaise Pascal de Clermont-Ferrand dans les Actes du colloque Kluge de février 2012 : Eric Lysœ et Vincent Pauval (éd. scient.), Alexander Kluge et la France. Pour une levée en masse de la narration.

9. - Das Labyrinth der zärtlichen Kraft, p. 589, p. 338.

10. - Christina Nord, « Der Großintellektuelle », in : taz.de, 25. 04. 2008, http:// 
Où est le centre, criait rabbi Madies, l'eau dédaignée permet au faucon de poursuivre sa proie.

Le centre est peut-être le décalage de la question ${ }^{11}$.

Plus de trois cartes se superposent dans ce dispositif topologique, ni centre ni périphérie, autant de tracés du livre avec disque de Kluge, du Livre sédimenté de Babylone, des films-sphères d'Eisenstein, du jardin, du nuage d'Internet... ${ }^{12}$

\section{Chambre d'échos}

Le jardin en enclos s'involue-t-il en chambre d'échos ? Tel serait le DVD, la poursuite du cinéma analogique d'auteur par les moyens du film numérique d'auteurs, à travers la télévision analogique, numérique d'auteur. L'intégrale des films cinématographiques de Kluge jusqu'en 1986, publiée en 2007, fait résonner non seulement des essais télévisuels et des films-minutes en affinités électives de lettres-images, des lectures littéraires d'auteur de sa voix « acousmétrique », infratextuelle, métatextuelle en écho, mais aussi les textes/images PDF de ses livres-films en flux autonomes, pourtant confluents. Ainsi l'espace littéraire se met-il en expansion à travers des typographies en noir et blanc ou en couleurs, constructivistes, dadaïstes. Le temps cinématographique s'incurve en ce Gesamtverbund de Gesamtkunstwerke, s'injectant les making of, aujourd'hui reflet sans cesse amplifié des films hollywoodiens ${ }^{13}$.

Une créature qui souffre change de forme, tel l'éléphant qu'on électrocute, et tout l'univers est décrit à partir de là, Youtube renvoyant les films-minute qui naguère s'additionnaient chez Kluge, la voix d'Ovide portant jusqu'à Youtube. Le programme en DVD du film cinématographique Les artistes sous le chapiteau : perplexes se constelle en voûte de trapéziste, « au-dessous, c'est le vide », de l'exploit inouï « 5 heures de Parsifal en 90 secondes », de lectures préméditées d'Alexander Kluge extraites du livre Les Artistes sous le chapiteau : perplexes, du bonus dénaturé et colorisé de l'antique film-minute Exécution d'un éléphant, et du commentaire hypertextuel en écho précurseur de Georg

www.taz.de/1/der-grossintellektuelle/type $=98$.

11. - « Wo ist das Zentrum? / schrie Rabbi Madies, / Das verschmähte Wasser erlaubt es dem Falken, / Seine Beute zu verfolgen / Das Zentrum ist vielleicht die Verschiebung der Frage. » Das Labyrinth der zärtlichen Kraft (n. 8), p. 340.

12. - $C f$ l'entretien d'Alexander Kluge avec Vincent Pauval qui précède cette étude, « Die Augen der Anderen ».

13. - Alexander Kluge, Sämtliche Kinofilme, Frankfurt a.M., Zweitausendeins, 2007. 
Stanitzek, qui rappelle que tout Kluge n'est que commentaire ${ }^{14}$. En commentaire critique de commentaire, Jan Philipp Reemtsma met au jour la tension entre les théorèmes et les exécutions textuelles de Kluge. La confiance originelle de l'éléphant, Urvertrauen, ne serait-elle pas le pur produit d'une civilisation de l'électricité et du premier cinéma, qui laisse tourner là un snuff movie en un emboîtement sanglant de sourdes lacunes ${ }^{15}$ ? C'est un change généralisé de formes en actes, « Gestaltänderung ». Par éléments, points, intensités, les sentiments affluent-ils dans les abysses ? La guerre de tranchées de 1914/18 ne cesse d'en ressurgir, s'arquant contre la voie lactée des états de choses et des données numériques qui nous submergent, au risque de la perte de toute orientation. Ne nous faudrait-il pas alors tenir des cartes géographiques de la subjectivité entêtée, pour esquiver cette omniscience de GPS ? Dès lors, l'internet bruirait selon Kluge « de la facticité surpuissante contre laquelle nous nous défendons, disons depuis Verdun en $1916^{16}$. »

\section{Eaux vives, mortes eaux}

Le film de Kluge L'assaut du présent contre le restant du temps, Der Angriff der Gegenwart auf die übrige Zeit (1984), risquait de nous laisser longtemps en suspens dans ce présent obstiné, puisque les médias numériques auraient aboli le futur, et que les attentats du 11 septembre 2001 auraient figé, selon Douglas Rushkoff, le compte à rebours du futur par une « digiphrénie » généralisée. Les médias de flux, flow media, par exemple twitter, paralyseraient les médias de sédimentation, stackes media, par exemple le courrier électronique. Mais Kluge ne déjoue-t-il pas cette opposition en son flot figé, multipliant les métamorphoses des films-minutes en films de dix heures, et inversement, par récursions réfléchissantes ? Pourtant, l'effectivité s'est elle aussi épaissie à ce moment crucial, a connu une montée en charge de matière dense ${ }^{17}$. Le flux entre les lettres et les images s'est intensifié pour parer au danger de la dé-réalisation, Entrealisierung. Ce retrait de réalité opéré par l'attentat contre les Twin Towers aurait déchiré l'effectivité,

14. - Ibid., DVD 3.

15. - Jan Philipp Reemtsma, «Urvertrauen in die Poesie ist das höchste Zivilisationsprodukt », $F A Z, 6$ janvier 2004, n 4, p. 33. L'éléphant est l'emblème cinégraphique, iconique d'Alexander Kluge. $C f$. par ex. "Eingemachte Elefantenwünsche », Neue Geschichten Unheimlichkeit der Zeit, op. cit., p. 202-206.

16. - «Insofern ist das Internet nur ein Bild für eine übermächtige Faktizität, gegen die wir uns etwa seit Verdun 1916 in Gegenwehr befinden. » A. Kluge in : FAZ 14. 01. 2010 „Gärten anlegen im Daten-Tsunami“ ( $c f$. note 8).

17. - «Die Wirklichkeit hat sich verdickt, hat sich materiell aufgeladen. » A. Kluge in: Die Zeit 23. 10. 2003, n ${ }^{\circ} 44$, „Der Friedensstifter“, Interview von Ulrich Greiner und Gesprächsführung Iris Radisch, <http://www.zeit.de/2003/44/L-Kluge/ komplettansicht?print=true> 
à l'encontre d'une guerre, cette modalité de réalité, Realitätsmodus ${ }^{18}$. Une brève histoire recueillie dans l'album de textes et d'images Seen sind für Fische Inseln expérimentera sur le mode ludique se transportant en Chine, une restauration «densifiante », mais filmique, de la réalité par le réalisateur Long Cauxu dans son film contre le terrorisme : « Confection de film après le 11.09.0119 ». Mais c'est l'un des premiers «magazines » télévisés d'Alexander Kluge, La Tour Eiffel. King Kong et la femme blanche - Der Eiffelturm. King Kong und die weiße Frau, diffusé en 1988, qui s'avérera anticipation du 11 septembre, restitué à l'image, à la lettre, par sa Fernseh-Nachschrift, sa transcription du $n^{\circ} 4$ de Facts \& Fakes en 2002, faite d'explosions en série et d'éclairs héraclitéens. Si les Twin Towers et la Tour Eiffel ne sont pas superposées dans ce fascicule, Kluge ne nous en disait pas moins en décembre 2002 : «Il s'agit d'une fantaisie sur les Twin Towers $20 »$. Plus tard, en chemin vers la nouvelle sphère publique Internet, la ruine du World Trade Center avoisine la fin des étoiles et l'affliction de la magicienne Alcina dans le moyen-métrage de 2007, rare Spielfilm de 49 minutes, Der Zauber der verdunkelten Seele, La magie de l'âme obscure. Nous sommes alors témoins d'une confusion créatrice entre Seele, l'âme, ce qui émane des eaux vives, et les mortes eaux des salles obscures, verdunkelte Säle, un Hörfehler d'où surgit le « nouveau film²1».

\section{Materia prima}

$\mathrm{Ne}$ faudrait-il pas une rébellion de la littérature contre l'universelle connexion de la toile, par une nouvelle clôture de l'action romanesque, demandent à Kluge ses interlocuteurs du Spiegel en 2008. Et Kluge de répondre : «On combine les histoires écrites au crayon, qui toutes ensemble donnent un catalogue des sentiments, plus tard à l'ordinateur ${ }^{22}$. » Ainsi son écriture endure-t-elle la différence tactile entre la main subjectile à la mine de plomb où se concentre toute l'énergie, et

18. - Sur la « disparition » et la « réapparition » de la réalité le 11 septembre 2001 selon Alexander Kluge, $c f$. Klaus Theweleit, Der Knall : 11. September, das Verschwinden der Realität und ein Kriegsmodell, Frankfurt a. M. / Bâle : Strœmfeld / Roter Stern, 2002, p. 158-166 («Alexander Kluge. King Kong und die Wirklichkeitsgarantien »).

19. - Alexander Kluge, Seen sind für Fische Inseln, Fernseharbeiten 1987-2008, Frankfurt a.M., Zweitausendeins, 2009.

20. - Alexander Kluge, «Ainsi s'en retourne l'écho », entretien d'Alexander Kluge avec Kza Han et Herbert Holl, in Herbert Holl et Günter Krause (dir.), Heiner Müller et Alexander Kluge arpenteurs de ruines, Paris, L'Harmattan, 2004, p. 171-185.

21. - Album de Seen sind für Fische Inseln, p. 185.

22. - « Kombiniert werden die mit Bleistift geschriebenen Geschichten, die zusammengenommen einen Katalog der Gefühle ergeben, dann später am Computer. » (« Ich liebe das Lakonische », entretien d'Alexander Kluge avec Mathias Schreiber et Volker Hage, Der Spiegel, n45, 2000, p. 336-340, cit. p. 340). 
le sujet-objet-projectile de la machine-médium de convergence se dissipant en nuage numérique, proches jusqu'à se toucher sur le site klugien de la Friedrichstraße ${ }^{23}$. Les Geschichten vom Kino de 2007, qu'il aura écrites, réécrites au crayon de bois, assis à son immense table ronde face à l'antique siège vide, reversent dans les lettres noires de la page blanche du « principe cinéma », cet immortel qui nous meut, tout l'art cinématographique des chambres noires et des bancs de montage numériques. Ces « singulières boucles vidéo-acoustiques-dialogiques » sont-elles dès lors littéraires de part en part, comme le pense Fritz J. Raddatz ${ }^{24}$ ?

Or, pour Kluge, raconter toutes ces histoires, projeter toutes ces images et lettres en mouvement, c'est comme poser une question en fulgurances de monades et de cartographies narratives où se frotteraient les plaques de subduction d'expériences « comme il y a des tremblements de terre. » Dès lors, demandent ses interlocuteurs du Zeit, narrer des multitudes de catastrophes, est-ce « éviter la prochaine catastrophe ? " « Non », réplique Kluge, « vous êtes trop radicaux sur ces questions. Ce que je fais est en deçà du seuil de la littérature. C'est de l'anti-littérature »25. De même que l'art cinématographique remonte à sa matière première, les lettres, de même les lettres remonteraient à la «matière première de la littérature ». En réécrivant, refilmant inlassablement Description de bataille. L'édification organisationnelle d'un malheur, Schlachtbeschreibung. Der organisationelle Aufbau eines Unglücks, avant 1989, après 1989, Kluge aurait reconduit la fiction documentaire à cette materia prima en deçà de l'opposition - due selon lui au hasard de la bifurcation fatidique entre Méliès et Lumière à l'aube du XX $\mathrm{X}^{\mathrm{e}}$ siècle - de la fiction et du document, à ces météores où s'éprouve toute expérience ${ }^{26}$.

Quelle résistance le cinéaste doit-il alors opposer à l'équarrissement hygiéniste contemporain, à la « convention collective de l'œil », alors que le film tend à quitter le cinéma pour les galeries et les musées? Le « principe cinéma » n'est-il pas en quelque sorte immortel de par ses lacunes mêmes, tant que sera cinématographique un film « dont le sujet peut se restituer en deux mots », comme l'exigeait Eisenstein de

23. - Quant au projectile klugien, $c f$. Kza Han, « Deux poèmes projectiles », et Herbert Holl, « La libellule comme projectile au futur antérieur d'Alexander Kluge », in Variations. Revue internationale de théorie critique, (en ligne), ${ }^{\circ} 18,2013$.

24. - « wundersame video-akustisch-dialogische Assoziationsschleifen », l'expression est d'Andreas Isenschmid, « Kluges Kapital », in : Die Zeit, $\mathrm{n}^{\circ} 50,4$ décembre 2008, p. 57. - Fritz J. Raddatz, « Zwischen Bluff und Verblüffung. Alexander Kluges Kino-Potpourri », in : Die Zeit, n²1, 16 mai 2007, p. 68.

25. - «Der Friedensstifter », entretien cité (n. 17) : «Kann das Erzählen vieler Katastrophen die nächste Katastrophe abwenden? » « Nein. Sie sind zu radikal in diesen Fragen. Was ich mache, ist unterhalb der Schwelle der Literatur. Es ist Antiliteratur. [...] ». 26. - Ibid. 
son film sur le Capital de Marx ? Regardant le Complexe-Allemagne de 2009, Kluge répond à celui qui lui demande si c'est encore là du cinéma : «Oui, aussi longtemps que nous sommes ici, dans ce cinéma ». Avec Eisenstein, on pourrait dire : aussi longtemps qu'est « cinématographique ce film qui se laisse restituer en deux mots ${ }^{27}$. »

À la fin du mois d'avril 2013, sur le grand écran de la salle Georges Franju à la Cinémathèque française, en présence d'Alexander Kluge, les neuf heures des Nouvelles de l'antiquité idéologique. Marx Eisenstein - le Capital, publiées en 2008, projettent lettres, images et nombres, en couleurs et noir et blanc, en format numérique, sans profondeur de champ, mais la dialectique de la surface et du noyau résiste obstinément, déployant ses variations discontinues et pourtant maintenues en contexture : à partir des simples « lettres » et croquis, materia prima du journal d'Eisenstein en vue d'un film sur le Capital de Marx jamais tourné, comment mettre en images, en sons, en textes le « grand poème » de Karl Marx paru en 1872 et les notations d'Eisenstein de 1928, sur le long chemin du retour vers l'antique terre ferme, de Marx à Ovide, en passant par Lénine, Engels ? Les Nachrichten aus der ideologischen Antike sont sous-tendues par le parallélogramme Joyce Eisenstein - Marx - Kluge. Comment filmer alors ce parallélogramme sous tension, se sont demandé ensemble Oskar Negt et Alexander Kluge, à la recherche de la materia prima infra-cinématographique, infra-littéraire d'une économie de la force de travail vivante. En quelles images le Capital est-il transposable, enregistrable?

Nulle réponse ne sera trouvée dans la dramaturgie sphérique des entretiens, des lectures de poèmes, des films brefs, des essais opératiques de ces trois DVD... Mais dans l'ultime séquence de la troisième partie, c'est le paroxysme joué par Helge Schneider « philosophant » longuement à coups de marteau sur un clavier non tempéré, le burlesque de ce condensé des Marx Brothers et de Dada, littéralement insoutenable, à mourir de rire dans la présence sans proche ni lointain de l'écran, en étalage des chiffres et des lettres, des images sans reflets. Das Zwerchfell erschüttern, dirait-on en allemand de la réaction des spectateurs, secouer la rate à la dilater. Or, en anatomie, Zwerchfell, c'est le diaphragme, ce muscle long, étroit entre poumons et estomac, d'où proviendra en français l'ouverture/fermeture du diaphragme photographique, cinématographique, en allemand Blende, l'illusion aveuglante. Cette Blende filme littéralement le Capital, à la lettre, condensant en quarante minutes la journée aveuglée de ce Capital, la longue méditation du Bloom de Joyce, se les insufflant de manière à la fois délirante et rigoureusement composée, désenchaînée telle la terre de l'Aurore de

27. - Gérard Mordillat, «Éloge du flou », MD septembre 2011,p. 27 ; Eisenstein, cité par Alexander Kluge, livret des Nachrichten, p. 13. 
Nietzsche. Le cinéma serait-il alors la « littéracie » de l'économie politique de la force de travail vivante ? C'est précisément le piano, l'instrument d'intérieurs bourgeois hyper-littérarisés, qui forme la basse continue de la résistance radicale de la lettre à sa numérisation à travers la prolifération de l'intérêt composé, surcomposé jusqu'à épuisement du temps et du Capital même. Tel est le Zinseszins, rejoué, déjoué sans fin par Helge Schneider en compositeur attitré des musiques d'Eisenstein, en destructeur de la gorgonéenne accumulation des marchandises immatérialisées en flux sur l'étal de l'écran.

Ainsi Helge Schneider, en bénéficiaire de Harz IV, incarnation de Marx et du compositeur déjanté d'Eisenstein, envoie-t-il par le sansfond, à bon droit, le «bon droit », Recht und Fug, de l'art, de l'économie, du fétichisme de la marchandise, mettant la main en plein dans le noir, cette cible sans queue ni tête. Der Griff ins Schwarze, c'est ainsi que s'intitule dans le long film multi-perspectiviste de six heures sur Dada la séquence qu'anime Bazon Brock, penseur de la ruine et du cas crucial, Ernstfall, avec Alexander Kluge, Dada et ce qui s'ensuivit, Dada und die Folgen : « Dada ist die Kunst des Unfugs », « Dada est l'art sans queue ni tête », c'est l'oxymore que démembre ici Bazon Brock ${ }^{28}$. À travers les performances de Hugo Ball, les manifestes de Tristan Tzara, les non-poèmes de Richard Huelsenbeck, c'est «l'insanité au corps », Unfug im Leib. Écrasé par la domination du «principe de croissance», de l'art contemporain, de l'économie financière et numérique, Dada ressurgirait aujourd'hui, selon Bazon Brock, à travers les capitales du trou noir, puisque la loi de croissance est mise au rebut par les règles d'une transformation perpétuelle. Sans se laisser croître, Dada s'était transformé souterrainement, transporté en Bulgarie, en Hongrie, après son envol public vers Paris et les Etats-Unis. Ce faisant, en citant, c'està-dire en appelant par leurs noms lettres, images dadaïstes en arrièreplans et incrustations, Brock et Kluge transforment leur film-essai en Spielfilm, comme Schiller parlait de Spieltrieb, affirmant tous deux « la normalité du contrefactuel ». " Gorgonisez-vous ! , tel est le mot d'ordre en Unfug repris de Dada. Les consonnes qui, dans les films, se regardent hors les voyelles pour revendiquer leur autonomie, c'est la Gorgone qui se réfléchit dans le bouclier de Persée, la brosse immaculée d'une femme qui se défléchit dans la brosse enchevêtrée de son mari, affirmant la «transcendance intramondaine » de toute lettre, l'illumination profane qui troue toute continuité. La trinité de cette bouche en infralangue s'accomplissait dans l'extase byzantino-dadaïste de Hugo

28. - Alexander Kluge, Der Griff ins Schwarze / Bazon Brock über Dada und die Folgen, DCTP 2012. Dès le 14 juillet 1916, Hugo Ball proclamait dans son manifeste inaugural : « Ich will meinen eigenen Unfug, und Vokale und Konsonanten dazu, die ihm entsprechen.» 
Ball qui, selon Bazon Brock, fut, non pas successivement dadaïste, puis extatique, d'abord auteur de manifestes, puis d'autobiographie, en Fuite hors du temps, Flucht aus der Zeit, mais l'un et l'autre, simultanément. En cette extase s'accomplit la «Vermüllung », pure déchéance de déchet, de ce qui s'ingère en matière d'art, d'ores et déjà mort. Typographie et parlure fusionneront en Dada, le rare surgissement de la Mündlichkeit, de l'oralité entre Bazon Brock et Kluge dénote une fois encore la proximité entre le sub-ob-projectile klugien dans sa latéralité et le pur projectile dadaïste reçu en pleine face par Walter Benjamin dans L'œuvre d'art à l'ère de sa reproductibilité technique.

\section{«Tant de commencement jamais ne fut »- «So viel Anfang war nie »}

Le livre-film de vie d'Alexander Kluge semble être un livre du commencement, du recommencement. Dans ses films de la documentation Le dernier été de la RDA de 2009, avec Cassian von Salomon, Kluge évoque son nouvel élan, lorsque vingt années auparavant, comme s'effondrait le mur de Berlin, les humains échappant à leur infra-sphère publique, Unteröffentlichkeit (Uwe Tellkamp), allaient dans la fièvre à la rencontre d'un futur antérieur, et que décembre 1989, le mois inconnu, se conjuguait en mode historique subjonctif... « En 1989 », dira-t-il en 2003, «j'ai pensé que nous allions vers un temps de féli-

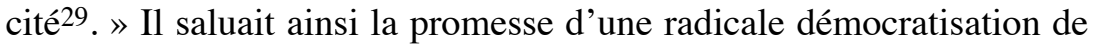
la nouvelle RDA : «Tant de commencement jamais ne fut ». Deux ans auparavant, il avait mis en grand sommeil ses films de cinéma, longs métrages et documentaires, ses livres et ses livres-films, pour fonder la DCTP avec ses innombrables films télévisuels à venir, échappant à la pesanteur d'un cinéma institutionnalisé, semi-déserté, harcelé par les bureaucraties. Les « travaux télévisuels » qui s'ensuivirent furent recueillis de 1987, l'année fondatrice, à 2008, l'année du complexe filmique Nachrichten aus der ideologischen Antike, dans les lacs qui sont îles pour les poissons : Seen sind für Fische Inseln. Ces œuvres télévisuelles d'Alexander Kluge paraissaient encore chez l'éditeur alternatif Zweitausendeins, tandis que le film inaugurait la filmedition de Suhrkamp. Mais en Allemagne, n'y a-t-il pas toujours tournant dans le tournant? «On dit : chaque révolution allemande, celle de novembre 1918 et celle de novembre 1989, ne va jamais plus loin que

29. - In : «Der Friedenstifter », entretien cité (n. 16). - Der letzte Sommer der DDR, Spiegel TV Dokumentation \& Alexander-Kluge filme, 2009, dctp, DVD 4, $\mathrm{n}^{\circ} 12$, Der Turm, entretien entre Uwe Tellkamp et Alexander Kluge. 
la veille de Noël, Heiligabend. Décembre 1989 apporte le tournant dans le tournant. Plus rien ne sera comme en décembre ${ }^{30}$. »

" J'observe à présent que cela s' "atavise" depuis 2001. L'époque devient atavique », dira Alexander Kluge en 2003 à ses interlocuteurs $\mathrm{du} Z \mathrm{Zit}^{31}$. Trois ans après encore, alors qu'il vient d'achever le chapitre grec de son Quint Livre, avec les guerres des Balkans à l'esprit et sous les yeux, il se demande si le siècle ne déraille pas, si nous ne revivons pas 1912, l'année du naufrage du Titanic, quelque veille prérévolutionnaire de cette Première Guerre mondiale qui commença le sanglant demisiècle. Est-ce la fin de la Grèce de la grande utopie, de Winckelmann, d'Iphigénie, mais aussi le nouveau commencement d'une mainmise allemande non sanglante, mais également inique ${ }^{32}$ ?

Cependant, l'année 1989 avait enlevé le deuxième clou que Lénine aurait planté dans le Capital de Marx, selon Dietmar Dath s'entretenant avec Kluge dans les Nouvelles de l'idéologie antique, alors que ce Capital tournait librement autour de son seul clou « primigène » telle l'aiguille d'une boussole. À présent, le Capital, le Capital et les capitaux évoluent derechef en toute liberté de mouvement et d'interprétation, de sorte que prolifère un « rhizome de pistes pour une lecture contemporaine » du Capital tandis que se joue son tragique «mélodrame planétaire 33 ».

Kluge va alors puiser dans la richesse littéraire du XVII ${ }^{\mathrm{e}}$ siècle afin d'endurer l'" inquiétance » médiatique du XXIe, traversant de l'une à l'autre de vastes zones temporelles désertiques. "Vous êtes terriblement solitaire parmi les vifs », lui dira-t-on. Solitude contemporaine paradoxale, puisque sont plus vifs que morts Heiner Müller, Einar Schleef..., qu'ils vivent encore « en esprits » avec lui. Et Kluge se fait fort de ramener Fassbinder à la vie en le «falsifiant » dans ses Kulturmagazine, à travers le kaléidoscope des images électroniques retravaillées en contexture. Mais étrangement, c'est Gottfried Benn, poète, essayiste scabreux, qui serait le plus vivant de tous en vertu de sa «nervosité moderne ». Dès lors, la phrase de l'histoire, l'image cinématographique, le mouvement de l'anti-opéra müllerien cherchent à atteindre un même cœur de cible : «Je suis un nerveux », dit Kluge,

30. - In: Begleitheft zur DVD « Der letzte Sommer der DDR / Die Wende in der Wende », 2009, non paginé.

31. - « Nun merke ich, daß es seit 2001 atavistisch wird. Das Zeitalter ist atavistisch. »In « Der Friedensstifter », entretien cité (n. 16).

32. - Adam Sobczynski, «Entgleist das Jahrhundert », in : Die Zeit, $\mathrm{n}^{\circ} 39$, 22 septembre 2011, p. 49 sq.

33. - Christophe Van Eecke, « Stock Footage and Shock Tactics. Eisenstein, Marx and Filming Capital», TRhu, Lokal 01, Breda 2009, p. 1-39, cit. p. 37 (« a rhizome of clues to contemporary reading of the book ») ; Jean Baudrillard, «Entretien sur la dualité des mondes », in : Rue Descartes, ${ }^{\circ} 49$, juin 2005, p. 68-81, citation p. 69. 
qui constate qu'à l'aune d'une blessure, toutes les perspectives, tous les horizons se modifient. "C'est ici que je dois faire mouche. En une image, en une phrase ${ }^{34}$.»

Déjà, le livre-film encore rudimentaire, Travail occasionnel d'une esclave, formulait cette ambition de lire « la quintessence d'un millier de pages ou de la capacité mémorielle hautement organisée de l'œuvre proustienne sous forme d'une histoire brève d'une à deux pages A4 tout au plus ${ }^{35}$. » Propager par concentrations d'histoires les processus d'apprentissage à issue mortelle ou vivifiante qui amènent « une autoorganisation de l'entêtement à l'encontre de son retardement », telle serait la puissance des œuvres de Hölderlin, Kleist, Proust, Joyce, Döblin, Kafka, Brecht, Marx, Freud... Il s'agit d'amplifier jusqu'aux dimensions d'Arachné la protestation, la chance insurrectionnelle d'un montage constellé.

\section{«Cinécriture »}

Que reste-t-il de la singularité de l'espace littéraire dans le Gesamtkunstwerk Kino d'Alexander Kluge ? Peut-être le bloc hiéroglyphique magique où le montage d'une image déchiffrable avec une écriture indéchiffrable met à l'épreuve cette déchiffrabilité de l'image ${ }^{36}$ ? C'est ce qui se produit chez Kluge lorsqu'il multiplie les nouvelles possibilités politiques de l'après-1989 en contre-productions d'une sphère publique qui ne cesse de se réengendrer tout en se décomposant à vie ${ }^{37}$. En contre-mouvement s'opère un démolissage d'artiste de l'espace public par les possibilités techniques d'installation du cinéma spatial avec multi-projections en décalages les unes avec les autres, Raumkino, qui après 1987 prit pour lui le relais du film de montage ou découpage, Schnitt- ou Montagefilm, et de l'essai télévisuel pur ${ }^{38}$. En 2007,

34. - « Ich bin ein nervöser Mensch, der feststellt, daß sich in dem Maß einer Verletzung sämtliche Perspektiven und Horizonte verändern. Das muß ich treffen. In einem Bild, in einem Satz. » In : « Der Friedenstifter », entretien cité.

35. - Winfried Menninghaus, « Geschichte und Eigensinn. Zu Hermeneutik-Kritik und Poetik Alexander Kluges », in Hartmut Eggert (dir.), Geschichte als Literatur: Formen und Grenzen der Repräsentation von Vergangenheit, Stuttgart, J.B. Metzler, 1990, p. 258-272, cité p. 263, Gelegenheitsarbeit einer Sklavin, Frankfurt a.M. : edition suhrkamp, SV, 1975, p. 221 (note) : « die Substanz von tausend Seiten oder des hochorganisierten Erinnerungsvermögens des Proustschen Werkes als Kurzgeschichte von höchstens ein bis zwei DIN-A-4 Seiten zu lesen. »

36. - Christina Scherer, «Das Bild der Schrift und die Schrift der Bilder», in : « Fernsehen ohne Ermäßigung. Alexander Kluges Kulturmagazine » (Augenblick. Marburger Hefte zur Medienwissenschaft, $\mathrm{n}^{\circ} 23$ ), p. 34-53, citation p. 38.

37. - Seen sind für Fische Inseln, p. 191, « Die Kategorie Öffentlichkeit », p. 191 $s q$.

38. - $C f$. le générique et l'argument hautement élaboré de l'installation, in: Seen sind für Fische Inseln, p. 186. 
l'année même des Geschichten vom Kino, Kluge donne à la Haus der Kunst de Munich Images multiples pour 5 projecteurs («Simultanés»). Boucle sans fin, dont la première eut lieu dans la Ehrenhalle de Hitler, aux murs et au plafond tendus de toile d'écran. Hans Richter, l'auteur de Combat pour le film et Ennemis du film aujourd'hui, amis du film demain, parus durant l'année fatidique de 1929, parcourt toute l'installation-éclair de vingt-trois minutes : « Ampex digital optics de 1987 à 1992 ». Parmi les titres en variations extrêmes repose «Die Bismarck auf dem Meeresgrund », paraissant incrusté sur un rouleau textuel en langue japonaise. L'image tant de fois publiée du mythique " géant cuirassé », invincible et pourtant envoyé par le fond au large de Brest en mai 1941 grâce à de petits avions-moustiques anglais, Moskitos, avant d'être enfin localisée en 1989, se contamine de l'indéchiffrabilité « pour nous » de cette «nouvelle histoire » datant de 1977 : «Le rythme temporel de l'abattage et celui de réparations. Un message non transmis », Der Zeitrhythmus des Abschlachtens und der von Reparaturen. Eine nicht übermittelte Nachricht ${ }^{39}$. La boucle sans fin de l'installation simultanée de 2007 est appelée par la boucle finale, à issue mortelle, de la nouvelle histoire ou anecdote de 1977, que son dénouement rend indéchiffrable, comme sa version japonaise de 2007 l'est de prime abord. Quelle est cette information non transmise ? En mortelle illustration de l'adage klugien "Es gibt immer einen Ausweg », une partie de l'équipage envoyé par le fond aurait rencontré dans la coque du navire une bulle d'air qui lui permettrait de survivre de mai jusqu'à la Saint Sylvestre 1941, plus longtemps que les plongeurs tirés vers le bas par leurs cloches, et plus longtemps que toute révolution allemande à ce jour. Du texte de 1977 qui déjà se réfléchit en auto-commentaire, à la traduction japonaise filmée et son hyper-commentaire dans l'installation murissent les raisins de la colère, mais aussi ces fruits de la confiance qui tombent à l'heure juste de l'arbre du texte profane, selon Walter Benjamin dans Sens unique. Mais dans le Simultané de 2007, le Bismarck est emporté par la tempête du " générateur d'aléatoire », Zufallsgenerator, en puissance d'une combinatoire aussi singulière qu'universelle ${ }^{40}$.

Les poèmes, ces monades temporelles indéchiffrables quant à leur sens originel, ressuscitent-ils alors dans ce cinéma klugien numérisé qui serait une sténographie de l'expérience, "Kurzschrift der Erfahrung $41 »$ ? Le montage, le banc numérique produisent-ils encore

39. - Neue Geschichten Unheimlichkeit der Zeit, Frankfurt a.M., Suhrkamp, 1977, p. 471-473.

40. - Ibid., p. 42. Walter Benjamin, Einbahnstraße, Gesammelte Schriften, éd. par Rolf Tiedemann, Frankfurt a.M., Suhrkamp, 1971 sqq., IV/1, p. 92.

41. - Christina Scherer, art. cité, p. 50. 
cette « dialectique instable de l'intuition et du concept », élargissant les possibilités de la littérature inscrite dans le film ${ }^{42}$ ? Kluge marquerait alors la renaissance de l'écriture dans le médium électronique. L'écriture klugienne de la main et de l'outil capte le DVD, qui semble à présent la happer à son tour par la grâce des albums contrapuntiques où se concentre le livre-film de jadis, égal en dignité, en intensité, en longueur, au film-livre, tels La Puissance des sentiments, La Patriote...

Recueillant une écriture autre, l'entretien klugien infini, audiovisuel-scriptural où chaque entretien est un film, chaque film un entretien, lui retire-t-il à son tour sa parenté avec la littérature ? À l'encontre de cette dernière, les thèmes et sujets se lèvent dans les motifs et les messages, Nachrichten. En même temps, les hypo- et hypertextes klugiens annonceraient-ils « la fin de la mort de la littérature », le retour d'un témoignage devenu objectif-subjectif, d'une nouvelle Zeugenschaft ${ }^{43}$ ? Parmi les grands témoins de Kluge, il en est un qui jadis l'appela «écrivain sans cœur », son contemporain depuis les années Kursbuch. Dès les Nachrichten aus der ideologischen Antike de 2008, plus intensivement encore dans Die Früchte des Vertrauens de 2009, Hans Magnus Enzensberger explore en face de Kluge les maximes de transsubstantiation de poèmes en messages-informations auditifs et visuels, par montage et superposition de la récitation et de la documentation épocale, ainsi de la vingt-troisième et de la vingt-cinquième de ses « Trente-sept ballades de l'histoire du progrès » du Mausoleum de 1975. En réponse à la question que pose Kluge dans Les Fruits de la confiance: « La destruction créatrice, ça existe ? », les huit minutes seize secondes du film «Bulle foncière de Paris » présentent d'abord la « visagéité » de haute destruction créatrice d'Enzensberger même, en fin connaisseur de Paris capitale du XIXe siècle ${ }^{44}$. Georges Eugène Hausmann (1809-1891), qui fit raser à Paris la maison même où il était né, fracassa Paris pour faire place nette, « enfumant les labyrinthes de la pauvreté » dès le début de cette vingt-cinquième ballade du Mausolée, « G.E.H » est cité à comparaître dans ce film sur le front de lignes où s'éprouve l'Urvertrauen klugien, en pure écriture musicale multicolore d'abord, par la seule voix de Kluge ensuite, dans la superposition des lettres de la ballade d'Enzensberger, par la voix du lecteur Kluge enfin. En splendide solitude textuelle, pictogrammatique, l'écran s'illumine, en noir et blanc, d'un syntagme de la ballade d'Enzensberger, en français dans le texte : «artiste démolisseur ». Mais cette appellation, cette

42. - Ibid.

43. - Stuart Moulthrop cité par G. Stanitzek, in : Essay - BRD, Berlin : Vorwerk 1,2011, p. 312.

44. - In: Alexander Kluge, Früchte des Vertrauens, filmedition suhrkamp, 2009, DVD III, ${ }^{\circ} 23$, « Grundstücksblase Paris ». 
interpellation du préfet Hausmann suscite en un suspens de toutes les couleurs de lettres et d'images une illumination profane. Détruisant ces images au fur et à mesure qu'ils les créent, Enzensberger et Kluge se retrouvent une nouvelle fois en conjonction des lettres du poème, des phrases-mondes de l'essai-conversation, des toiles en contextures réticulées d'Arachne ${ }^{45}$. En vertu de ce témoin oculaire, auditif, multisensoriel affûté et endurant, Enzensberger à la subjectivité libérée de toute intériorité romantique, Kluge essaie ici de libérer la lettre de sa pureté littéraire, à l'instar de son cinéma ${ }^{46}$. Dans ces ballades d'Enzensberger, la dialectique lyrique secoue la " grille de la prose-monde », tel «le procès verbal d'un bruit blanc dans la tête ${ }^{47}$.

45. - Sur ces « tissages » klugiens, $c f$. Thomas Elsaesser, « Mélancolie et mimétisme : les énigmes d’Alexander Kluge », in Trafic, n³1, automne 1999, p. 70-94.

46. - Norbert Bolz, « Eigensinn. Zur politisch-theologischen Poetik Hans Magnus Enzensbergers und Alexander Kluges », in : Jochen Hörisch et Hubert Winkels (dir.), Das schnelle Altern der neuesten Literatur. Essays zu deutschsprachigen Texten zwischen 1968 und 1984, Düsseldorf, Claassen, 1985, p. 40-59, citations p. 40, 41.

47. - Ibid., p. 42, 44 : « wie das Protokoll eines Rauschens im Kopf». 
